\title{
Usability of building information modeling (BIM) for generating virtual geographic environments (VGEs)
}

\author{
Ondřej Kvarda ${ }^{\mathrm{a}, *}$ \\ ${ }^{a}$ Department of Geography, Faculty of Science, Masaryk University, Brno, kvarda.ondrej@mail.muni.cz \\ * Corresponding author
}

Keywords: virtual geographic environment, VGE, building information modeling, BIM, 3D modeling, conversion

\begin{abstract}
:
In general, the article deals with the possibility of connecting spatial information from the fields of architecture and geoinformatics. BIM is a commonly used digital representation of physical and functional characteristics of a building and its components throughout the building's whole life cycle with the focus on large-scale details that finds its application in the fields of architecture, civil engineering, and construction or project management (Gu, London, 2010; Volk, 2014). If more and more public buildings nowadays already have their BIM digital models, is it possible to take over these models and effectively repurpose them into virtual environments applicable in virtual reality where for example fire drills could be realized without the need to use the premises of real buildings?
\end{abstract}

In practice, this article concerns the conversion of a 3D architectural building model in the IFC data format (a standard data format for BIM) from BIM/CAD software Autodesk Revit to game engine Unity, its subsequent modification, and creation of an environment as faithful to its real-life counterpart as possible. The created virtual environment can then be used to manage experiments, spatial analyses, and case studies.

The process described in this article included the following steps: Conversion of the selected building model to a data format supported by Unity and its import to said software. Rearrangement of a complex and often nonuniform hierarchy of imported building elements and elimination of objects with far too detailed geometry or redundant objects that would not be accessible by the user in the final version of the model. Fixing basic construction elements that did not correspond with their real form or were needlessly fragmented into several separate objects. Editing out geometric and topological errors produced by the conversion process. Selection of relevant objects that were to be included in the environment. Modeling the interior (furnishing, plants, signs, doors, etc.) and exterior of the building based on their real counterparts so that the virtual objects look as similar as possible to the real ones. And the lighting of the scene along with the final adjustment of some objects geometry where needed.

The final virtual environment was then utilized in an application build intended for use in virtual reality and was part of a study dealing with the comparability of experiments in real and virtual environments with a focus on wayfinding and locomotion where an emergency evacuation took place in both the real and virtual environments of the building and chosen parameters were then measured and evaluated.

Potential possibilities regarding optimization and limits of the current presented solution are discussed as well.

\section{imported BIM model}

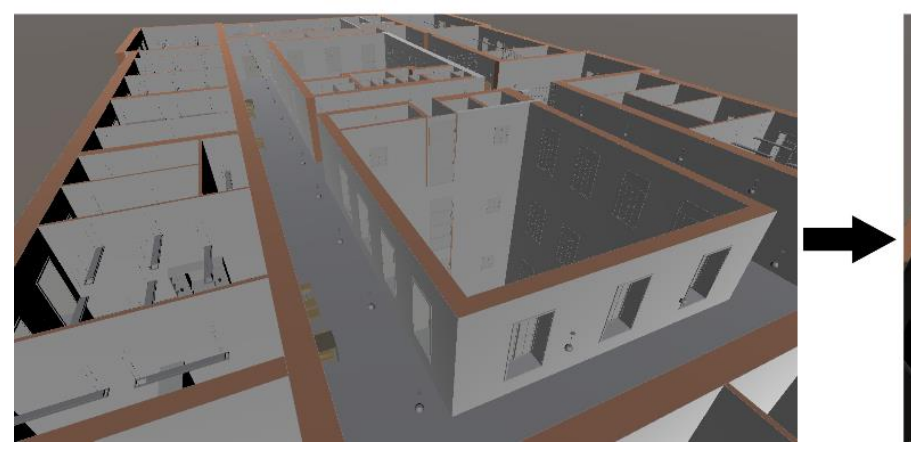

created VGE

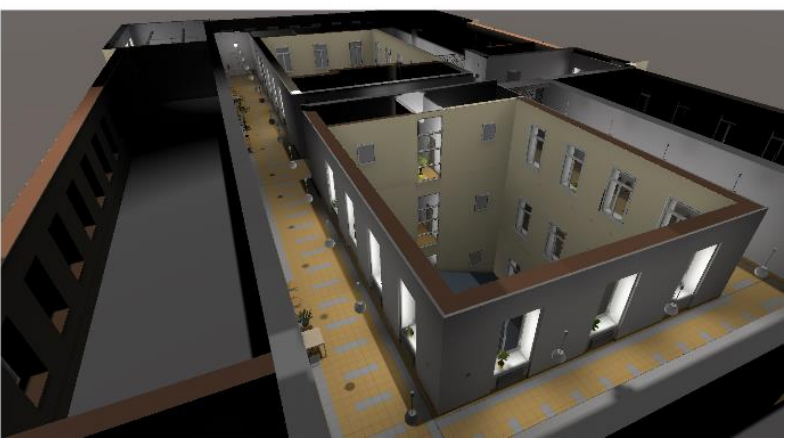

Figure 1. Difference between the imported BIM model of the building and the finished VGE in game engine Unity 